\title{
State and Local Health Agency Engagement in HIE: A Cross-Sectional Survey
}

\author{
Brian E. Dixon ${ }^{\star 1,2,3}$, Roland E. Gamache ${ }^{1,2}$ and Shaun J. Grannis ${ }^{4,2}$ \\ ${ }^{1}$ School of Informatics, Indiana University, Indianapolis, IN, USA; ${ }^{2}$ Regenstrief Institute, Indianapolis, IN, USA; ${ }^{3}$ Center of Excellence \\ on Implementing Evidence-Based Practice, Department of Veterans Affairs, Veterans Health Administration, Health Services \\ Research and Development Service, Indianapolis, IN, USA; ${ }^{4}$ Indiana University School of Medicine, Indianapolis, IN, USA
}

\section{Objective}

To characterize state and local health agency relationships with health information exchange organizations.

\section{Introduction}

There is growing interest in leveraging available health information exchange (HIE) infrastructures to improve public health surveillance (1). The Health Information Technology for Clinical and Economic Health Act and Meaningful Use criteria for electronic health record (EHR) systems are among the factors driving the development, adoption and use of HIEs. HIEs deliver or make accessible clinical and administrative data as patients are admitted, discharged, and transferred across hospitals, clinics, medical centers, counties, states and regions (2). While several HIE infrastructures exist (3), there is little evidence on the engagement in HIE initiatives by state and local health agencies.

\section{Methods}

An online survey of state and local health officials was conducted in six states where HIEs were known to be present. Half of the states were funded by the Centers for Disease Control and Prevention (CDC) to engage public health agencies in HIE activities; the other half received no such funding. A total of 143 officials were invited to participate; 73 (51\%) responded. The survey asked respondents about their agencies awareness, engagement, and data exchange with HIEs. The survey further asked agencies about their perceptions of barriers and challenges to public health engagement with HIE organizations.

\section{Results}

Just $25 \%$ of agencies had a formal relationship, typically created through a memorandum of understanding or data usage agreement, with at least one nearby HIE. The majority (54\%) of agencies either had no relationship $(20 \%)$ or only an informal relationship $(34 \%)$ with an HIE. The remaining agencies (18\%) reported that no HIE existed in their jurisdiction. Agencies in states that had received CDC funding for HIE engagement were more likely (14 versus 2 ) to be formally partnered with an HIE.

\section{Conclusions}

Few public health agencies are formally engaged in HIE. Financial costs, human resources, and concerns regarding privacy/security were the top cited barriers to broader engagement in HIE. For public health to be an active participant in and reap the benefits of HIE, greater investment in state and local public health informatics capacity, including human resources, and education regarding HIE privacy and security practices are needed.

\section{Keywords}

Health Information Exchange; Electronic Laboratory Reporting; Public Health Surveillance; Public Health Informatics

\section{Acknowledgments}

This work was supported, in part, by the Indiana Center of Excellence in Public Health Informatics through a grant from the CDC (501HK000077).

\section{References}

1. Savel TG, Foldy S. The role of public health informatics in enhancing public health surveillance. MMWR Surveill Summ. 2012;61:204

2. Dixon BE, Zafar A, Overhage JM. A Framework for evaluating the costs, effort, and value of nationwide health information exchange. J Am Med Inform Assoc. 2010;17(3):295-301.

3. eHealth Initiative. The State of Health Information Exchange in 2010 Connecting the Nation to Achieve Meaningful Use. Washington, DC2010 [cited 2010 September 29]; Available from: http://ehealthinitiative.org/uploads/file/Final\%20Report.pdf.

*Brian E. Dixon

E-mail: bdixon@regenstrief.org 